\title{
INVESTIGATION OF THE APPLICABILITY AND EFFICIENCY OF DIFFERENT MATHEMATICAL MODELING AND NUMERICAL SIMULATION METHODS FOR SOIL-TOOL INTERACTION
}

\author{
AndrÁs ZaChár, István KePpler And István OldAL \\ Institute of Mechanics and Machinery, Szent István University \\ Páter K. u. 1., H-2103 Gödöllő, Hungary \\ Oldal. Istvan@gek.szie.hu
}

[Received: September 18, 2015. Accepted: February 22, 2016]

\begin{abstract}
Dedicated to Professor Barna Szabó on the occasion of his eightieth birthday and to Professor Imre Kozák on the occasion of his eighty-fifth birthday
\end{abstract}

\begin{abstract}
Three different mathematical modeling and numerical simulation approaches the Finite Volume Method (FVM-CFD), Smoothed-Particle Hydrodynamics-Finite Element Method (SPH-FEM) and the Discrete Element Method (DEM) - are used to predict the draft forces of a plow moldboard interacting with cohesive granular material. In situ draft force and pressure field measurement data and simulation results are compared to investigate the efficiency of the applied mathematical models and numerical methods to predict the behavior of the soil flow around the studied plow moldboard. In the case of SPH-FEM modeling threedimensional explicit dynamic computations have been carried out in AUTODYN ANSYS Workbench 12 environment. The soil has been modeled as SPH solid body. Failure behavior of the soil has been described with the Drucker-Prager model. In the case of DEM modeling, the soil has been described as an assembly of large number of individual particles. For modeling the mechanical behavior of the soil, the Hertz-Mindlin with bonding contact model was used. The micromechanical parameters describing the granular assembly have been determined by a calibration method based on the standard shear test. In the case of FVMCFD modeling a three dimensional, multiphase flow computations have been carried out in the Ansys CFX 11 environment. The developed soil stress and pressure field on the surface of the plow moldboard and the induced draft force have been studied to gain more information about the entire physical process. The rheological behavior of the soil has been characterized as a Bingham plastic model. Comparison of the efficiency of different simulation approaches has been carried out to gain information on the efficiency of the different mathematical modeling techniques and the numerical calculations.
\end{abstract}

Keywords: Soil-tool interaction, finite volume method, smoothed-particle hydrodynamics method, discrete element method

\section{NomenClature}

Latin symbols

$A(\mathbf{r}) \quad$ arbitrary scalar or vector field of a physical quantity (SPH)

$\langle A(\mathbf{r})\rangle$ approximation of the field variable $(\mathrm{SPH})$

(C)2016 Miskolc University Press 


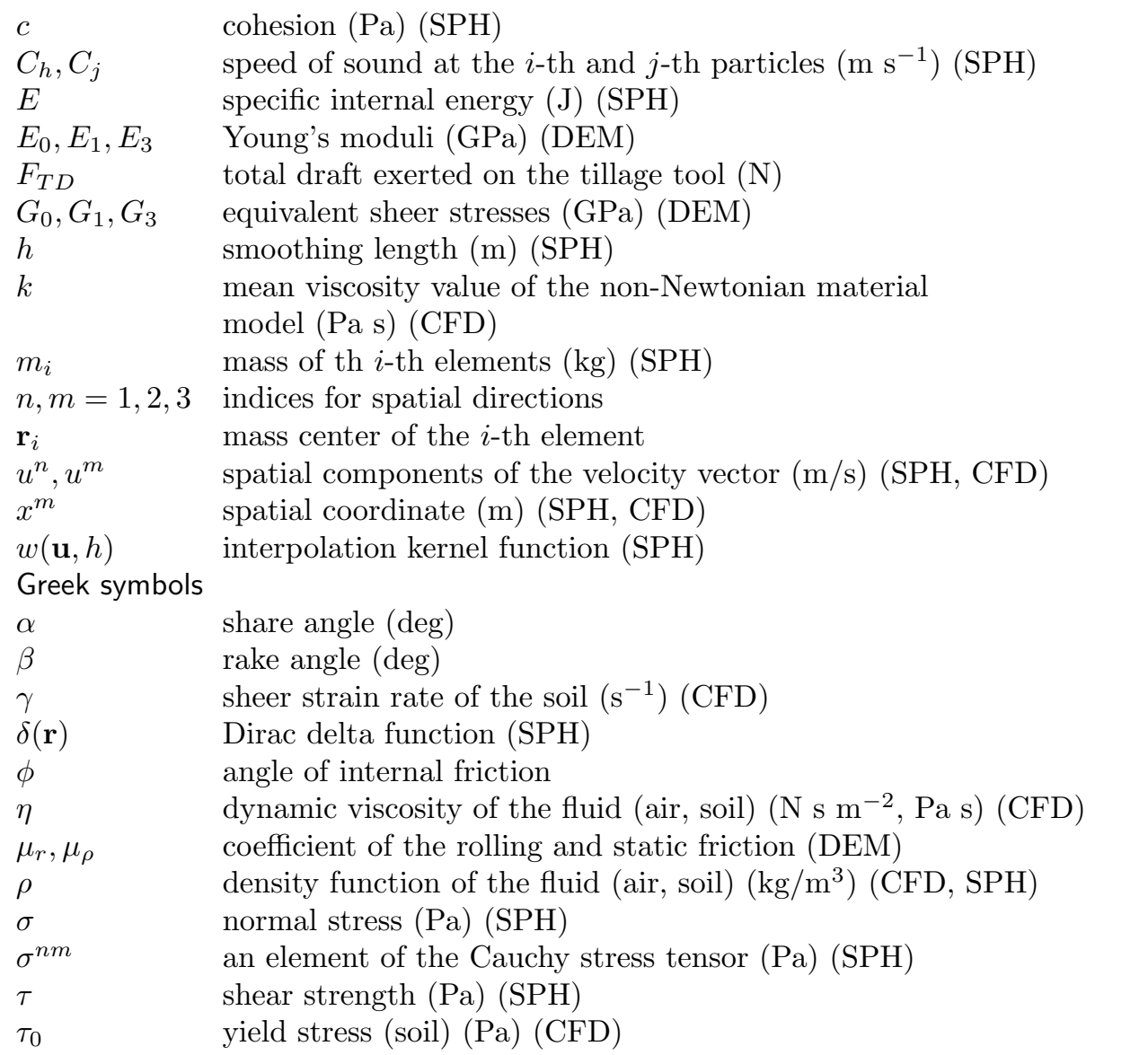

\section{INTRODUCTION}

The study of tillage tool interaction on different types of soil is an important phase of tool design and development. The total draft of a tillage tool is an important factor of the overall efficiency of soil tillage. Moldboard plowing is one of the most important and most energy consuming processes of agricultural production; for this reason adequate knowledge on draft forces and the developing pressure field on the surface of the tillage tool is necessary to improve the efficiency of the design process. Until now large amount of research has been conducted to study the dependency of draft force on the soil properties and tillage tool geometry. Generally three different properties are used to characterize the efficiency of a tillage tool, according to Karmakar and Kushwaha et al. [1]. These are the draft to operate the tillage tool - in this case the plow - the soil loosened and the total energy requirement. Several different attempts have been carried out in the last couple of decades to develop an analytical description of the soil tool interaction and formulation of the draft force. According to Godwin's review 2] the following two components of the soil forces must be taken into account: 
the horizontal (draft) force that is required to pull the tool through the soil and the vertical force, which assists or prevents the tool's penetration into the soil. These forces are the function of rake angle $\beta$, tine depth $d$, tool width $w$ and tillage velocity $v$. Most of the analytical predictions of draft forces acting on wide tines are based on the equation presented by Godwin and O'Dogherty 3$]$ which is sometimes referred as the Universal Earthmoving Equation

$$
F=\left(\gamma d^{2} N_{\gamma}+c d N_{c}+c_{a} d N_{c a}+q d N_{q}+\frac{\gamma v^{2}}{g} d N_{a}\right) w
$$

where $F$ is the draft force, $\gamma$ is the specific weight of soil, $g$ is the gravitational acceleration, $c$ is cohesion, $c_{a}$ is adhesion, $q$ is surcharge pressure and $v$ is the working velocity. $N_{\gamma}, N_{c}, N_{c a}, N_{q}$ and $N_{a}$ are dimensionless factors. According to Onwualu and Watts [4] the average deviation of the analytically predicted draft force from the experimental one varies from $6 \%$ to $160 \%$. The aim of this study is to test the applicability of different soil modeling methodology and numerical approaches simulating the soil moldboard plow interaction. This paper presents a comparison and evaluation of the studied mathematical models and numerical methods simulating a plowing process.

\section{Mathematical MODELS}

3.1. Main features of the two models considered. The mechanical behavior of agricultural soils is an extremely difficult area of research due to the complexity of soil parameters and operating conditions. Two basically different mathematical modeling approaches exist to describe the behavior of soil flow: these are continuum and discrete element modeling. The continuum model is based on the Eulerian modeling concept of the solid and fluid material assembled with infinitely small deformable and flexible cubes. The discrete element method is based on Lagrangian description, i.e., the mechanical interaction and motion of the individual particles is modeled.

3.2. Finite volume method (continuum modeling). Many different studies have shown that agricultural soils are approximately show brittle fracture mechanical behavior during soil tillage (see Chandler [5], Aluko and Chandler [6]). Several other studies hypothesized different type of soil mechanical behavior: the visco-elastic model has been used and studied by Kocher and Summers [7], the hypo-elastic model has been investigated by Rosa and Wulfsohn [8] and the elastic- perfectly plastic model has been tested by Mouazen and Neményi [9]. Soil failure and pressure patterns for flat cutting blades in soils of different strengths have been examined by Elijah and Weber [10] to gain more knowledge about the soil cutting blade interaction. Finite element modeling of the interaction of the cutting edge of tillage implements with soil has been conducted and the results compared with previously measured tillage forces (see Fielke [11]). In several different investigations the method of computational fluid dynamics ( $\overline{\mathrm{CFD}}$ ) has been used to model soil tillage tool interaction. Formato et al. 12 determine a virtual material able to quantitatively reproduce the distribution 
of pressure field on the working surface of a plow. Karmakar et al. 11 report a successful application of the Bingham plastic model to investigate the interaction between the soil and a flat tillage tool in a wide range of tool operating velocities. The physical problem is a steady, three-dimensional flow of a two-phase fluid where the first one is the air and the other one is the soil phase. The momentum equation of the fluid is based on the three-dimensional Navier-Stokes equations. A simple finite volume method is applied to solve the momentum and continuity equations. The dependent variables that describe the present flow situation are the pressure $P$ and the velocity components $U_{1}, U_{2}, U_{3}$ in the $x_{1}, x_{2}, x_{3}$ directions, respectively. The following set of partial differential equations for $U_{1}, U_{2}, U_{3}$ and $P$ as functions of $x_{1}, x_{2}, x_{3}$ describes the flow field around the moldboard plow in steady state flow condition.

(a) Continuity equation. By using tensorial notations in a Cartesian coordinate system for the continuity equation we can write

$$
\frac{\partial \rho}{\partial t}+\frac{\partial}{\partial x_{i}} \rho U_{i}=0
$$

(b) Momentum equations. The following equation system is the representation of the momentum equations in the Cartesian coordinate system:

$$
\frac{\partial}{\partial t} \rho U_{j}+\frac{\partial}{\partial x_{i}} \rho U_{j} U_{i}=-\frac{\partial P}{\partial x_{j}}+\frac{\partial}{\partial x_{i}}\left(\eta \frac{\partial U_{j}}{\partial x_{i}}\right)
$$

Here $\eta$ is the dynamic viscosity and $\rho$ stands for the density.

(c) Applied material models. Modeling the mechanical behavior of different soil types is a difficult task because of the extreme complexity of soil structure. Mechanical properties of different soil types depend on the soil texture, moisture content, soil compaction level and several other factors occurring in the field. The applied material model in the numerical calculations is the Bingham plastic model, which considers the cohesion effect of the soil. A Bingham plastic substance is a viscoplastic material that behaves as a rigid body at low stresses (below the yield stress). Above the yield stress the Bingham plastic material flows as a viscous fluid. Bingham plastic materials require two distinct parameters, the yield stress and the plastic viscosity. Equation (4) shows the mathematical formulation of the Bingham fluids:

$$
\gamma=\frac{\partial U_{i}}{\partial n}=\left\{\begin{array}{cc}
0 & , \quad\left|\tau_{i, j}\right|<\tau_{0} \\
\frac{\tau_{i, j}-\tau_{0}}{\eta}, & \left|\tau_{i, j}\right| \geqslant \tau_{0}
\end{array}\right.
$$

where $\tau_{0}$ is the yield stress of the soil, $\eta$ is the plastic viscosity and $\partial U_{i} / \partial n$ is the shear strain rate.

(d) Domain discretization and the applied computational grids. A schematic diagram of the plow indicating one of the applied grids for the computational domain is shown in Figure 1. The size of the calculation domain is chosen so that the wall effect can be neglected and some test calculations have been 


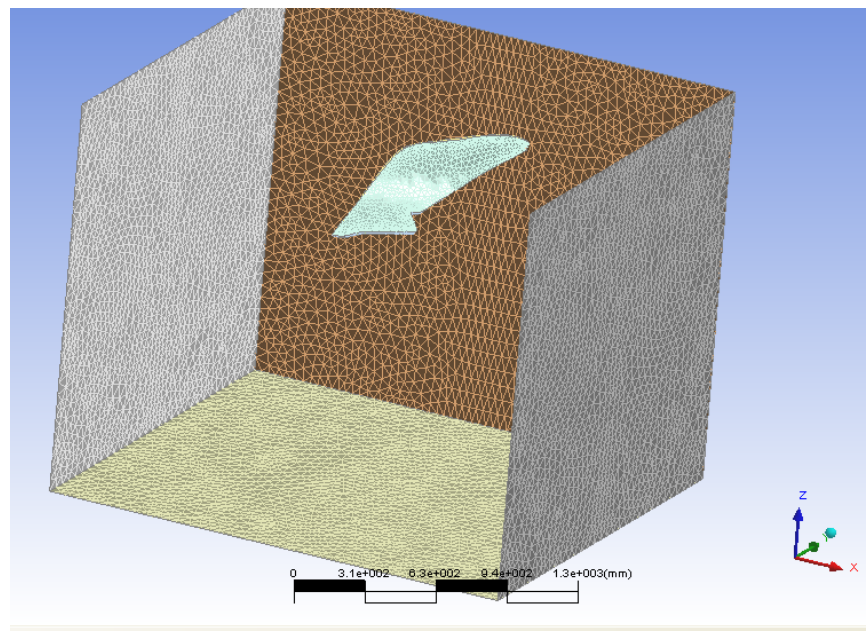

Figure 1. Computational domain

carried out to check the independence of the numerical result from the horizontal and vertical size of the domain. A careful check for the grid-independence of the numerical calculation has been created to ensure the accuracy and validity of the numerical calculations. Four different grids have been used to check the grid independence of the numerical scheme. The total draft of the plow has been applied to test the grid independence of the numerical calculations.

Table 1. Draft force and its relative error on the grids studied

\begin{tabular}{|l|c|c|c|c|}
\hline Studied grids & I. & II. & III. & IV. \\
\cline { 2 - 5 }$($ Number of cells) & 3325486 & 5916506 & 7317622 & 12591842 \\
\hline Draft force $F[\mathrm{~N}]$ & 4208.17 & 4191.27 & 4178.19 & 4176.81 \\
\hline $\begin{array}{l}\text { Relative error of the force } F \\
100 \mid \frac{F_{\text {coarser }}-F_{\text {finest grid }}}{F_{\text {finest grid }}}\end{array} \%$ & 0.750 & 0.346 & 0.033 & - \\
\hline
\end{tabular}

The generated grid is much finer near the leading edge of the plow-share than near the outer boundary of the computational domain. Comparison of the results of the calculation on the different grids is shown in Table 1 .

Relative error was calculated according to the assumption that the result of the finest grid is approximately ideal. The calculated results show that the difference between the induced draft forces on the finest grids is less than $0.03 \%$. This means that a grid resolution for the applied calculation domain finer than the third (III) grid is acceptable to produce physically realistic calculation results independent from the numerical grid.

(e) Multiphase flow computation, initial and boundary conditions. The investigated flow situation is a free surface flow problem which is a special case of 
multiphase flow computation where the fluids are separated by a distinct resolved interface. Both fluids (soil and air) share a common flow field. The working fluids are not mixed in the micro-scale rather they are mixed on a macroscopic scale with a discernible interface between the fluids. A homogeneous multiphase model has been incorporated into the flow simulation because the interface between the two fluids remains well defined and none of the dispersed phase becomes entrained in the continuous phase. The soil flow on the upstream side of the plow is subcritical and the downstream conditions are not known but can be estimated. Simulation of free surface flow usually requires defining boundary and initial conditions to set up appropriate pressure and volume fraction fields. Simulating the soil-tool interaction the following conditions are set. At the inlet boundary the volume fraction above the free surface is 1 for air and 0 for soil and below the free surface it is 0 for air and 1 for soil. Furthermore the height of the soil at the inlet and outlet locations is defined with $h_{\text {in }}=0.3 \mathrm{~m}$ and $h_{\text {out }}=0.3 \mathrm{~m}$. With the defined height of the soil in the computation domain the inlet, outlet and initial pressure distribution can be specified $P_{u p}=\rho_{\text {soil }} g h_{\text {in }}, P_{d}=\rho_{\text {soil }} g h_{\text {out }}$ where $P_{\text {up }}$ and $P_{d}$ are the upstream and downstream pressure at the inlet and outlet. The initial velocity field is zero everywhere in the calculation domain. A uniform, constant velocity profile is assumed at the inlet position. The gradient of the velocity profile is assumed to be zero at the end of the outlet location. A free-slip boundary condition has been applied at the bottom and the left and right wall of the computational domain. At the top of the calculation domain a free-surface boundary condition is specified. The initial values of the air and soil volume fraction are also supplied with an assumption that the virtual field contains a furrow before the plow in the appropriate location.

3.3. Discrete element method (discrete element modeling). By the use of Discrete Element modeling technique (DEM), the problem is solved by applying and solving the equation of motion on each individual particle of the bulk material assembly 13]. For modeling the mechanical behavior of the soil, EDEM has been applied as a discrete element software using "the Hertz-Mindlin with bonding" contact model [14]. In this model, the particles are glued with a finite sized bond. In the discrete element model from the beginning of the simulation until "turning on the bonded contacts" the simulation evaluates the contact forces according to the Hertz-Mindlin no slip contact model: the material and interaction parameters have their effect on the normal- and tangential forces. The normal force has the following form

$$
F_{n}=\frac{4}{3} E_{0} \delta^{\frac{3}{2}} \sqrt{R_{0}}-2 \sqrt{\frac{5}{6}} \frac{\ln C_{r}}{\sqrt{\ln ^{2} C_{r}+\pi^{2}}} \sqrt{2 E_{0}} \sqrt[4]{R_{0} \delta} \sqrt{m_{0}} v_{\text {nrel }}
$$

where

$$
\frac{1}{E_{0}}=\frac{1-\nu_{1}^{2}}{E_{1}}+\frac{1-\nu_{2}^{2}}{E_{2}}
$$


is the equivalent Young modulus of the two interacting soil particles and $\delta$ is the overlap between these two soil particles. This normal overlap represents the normal deformation of a particle. The normal overlap $\delta$ between two particles $i$ and $j$ at positions $x_{i}$ and $x_{j}$ (where $x_{j}-x_{i}$ is the distance measured on the line connecting the centers of the two overlapping particles) with radii $R_{i}$ and $R_{j}$ is defined as: $\delta=R_{i}+R_{j}-\left(x_{j}-x_{i}\right)$.

$$
R_{0}=\frac{R_{1} R_{2}}{R_{1}+R_{2}} \quad \text { and } \quad m_{0}=\frac{m_{1} m_{2}}{m_{1}+m_{2}}
$$

are the equivalent radius and mass while $v_{\text {rel }}$ is the relative velocity of the soil particles. The tangential force is given by the following equation

$$
F_{t}=-8 G_{0} \sqrt{R_{0} \delta} \delta_{t}-2 \sqrt{\frac{5}{6}} \frac{\ln C_{r}}{\sqrt{\ln ^{2} C_{r}+\pi^{2}}} \sqrt{2 G_{0}} \sqrt[4]{R_{0} \delta} \sqrt{m_{0}} v_{\text {trel }}
$$

where

$$
\frac{1}{G_{0}}=\frac{2-\nu_{1}}{G_{1}}+\frac{2-\nu_{2}}{G_{2}}
$$

is the equivalent shear modulus of the two interacting soil particles, $\delta_{t}$ is the tangential overlap between the two particles and $v_{\text {trel }}$ is the tangential component of the relative velocity of the soil particles. The tangential overlap is the tangential displacement of the contact point up to the point at which the contact ends or the particle begins to roll or slip. The tangential overlap represents the tangential deformation of a particle. The tangential force is limited by the Coulomb friction $\mu_{s} F_{n}$ where $\mu_{s}$ is the coefficient of static friction. The moment from rolling friction is $M_{r}=-\mu_{r} F_{N} R_{i} \omega_{i}$, where $R_{i}$ is the distance of the contact point from the centre of the $i$-th soil particle and $\omega_{i}$ is the unit angular velocity vector, which is a dimensionless quantity representing only the direction of rotation of the $i$-th soil particle. $\mu_{r}$ is the coefficient of rolling friction. The tangential force also has moment on the particle: $M_{t}=F_{t} R_{i}$. From $t>t_{\text {bond }}$ (from that time when bonding is "turned on", i.e., the particles are glued together) the bonding forces/moments acting on the particles are set to zero initially, and after that are incremented every time step:

$$
\delta F_{n}=-v_{n} S_{n} A \delta t, \quad \delta F_{t}=-v_{t} S_{t} A \delta t, \quad \delta M_{n}=-\omega_{n} S_{t} J \delta t, \quad \delta M_{t}=-\omega_{t} S_{n} \frac{J}{2} \delta t
$$

where

$$
A=R_{B}^{2} \pi, \quad J=\frac{1}{2} R_{b}^{4} \pi .
$$

$R_{B}$ is the radius of the bonding glue, $S_{n}, S_{t}$ are the normal and shear stiffness per unit area, $\delta t$ is the time step, $v_{n}, v_{t}$ are the normal and tangential velocities of the particles and $\omega_{n}, \omega_{t}$ are the normal and tangential components of the angular velocity vector. The bond is broken when the normal and tangential stresses exceed some predefined value:

$$
\sigma_{\max }<-\frac{F_{n}}{A}+\frac{2 M_{t}}{J} R_{B}, \quad \tau_{\max }<-\frac{F_{t}}{A}+\frac{M_{t}}{J} R_{B} .
$$

These bonding forces/torques are added to the standard Hertz-Mindlin forces. During the simulations, the linear and angular momentum theorem is used to write the 
equation of motion for all the individual particles, resulting in a large number of differential equations to be solved in a sufficiently large number of time steps. The applied time step has great impact on the stability of the numerical calculation. For the simulation $25 \%$ of the Rayleigh-type time step has been selected:

$$
\delta t=0.25 T_{R}=0.25 \cdot(0.1631 \nu+0.8766)^{-1} \pi R\left(\frac{\rho_{p}}{G_{p}}\right)^{\frac{1}{2}} .
$$

3.4. Smoothed-particle hydrodynamics method. The smoothed particle hydrodynamics method was originally invented by Monaghan and Gingold [15] for astrophysical applications. The SPH method has been successfully applied to several different impact problems since that, for example ballistic impact problems have been investigated by Hayhurst et al. [16], a pseudo-spring based fracture model of impact dynamics was studied by Chakraborty and Shaw [17], and it was applied to study complex fluid-solid surface interactions (see Yang et al. [18]). The SPH method has been found to be useful to study soil flow behavior and interaction with other bodies or fluids. Bui et al. 19] carried out a numerical simulation to investigate the soilwater interaction by the SPH method. Lu et al. 20] created a comparative study of a buried structure in soil subjected to blast load. SPH is effective and accurate at modeling material deformation as well as adaptable in terms of specific material models and besides to solve computational fluid dynamic problems, it can be also applied solving continuum mechanics problems with large deformations like machining problems. Calamaz et al. [21] studied the tool wear effect through a comparison between experiments and SPH numerical modeling of machining hard materials. Agricultural application is a new area of SPH application.

The basic concept of SPH is that the method does not use finite elements and mesh during discretization. SPH model contains only particles. These particles are not bodies (like discrete elements) but interpolation points (like nodes of a finite element). SPH involves the motion of a set of points. Points have masses and velocity and these values are known. Any field can be described with Dirac delta function:

$$
A(\mathbf{r})=\int A\left(\mathbf{r}^{\prime}\right) \delta\left(\mathbf{r}-\mathbf{r}^{\prime}\right) \mathrm{d} \mathbf{r}^{\prime}
$$

Function $\langle A(\mathbf{r})\rangle$ is an approximation of this field

$$
\langle A(\mathbf{r})\rangle=\int A\left(\mathbf{r}^{\prime}\right) w\left(\mathbf{r}-\mathbf{r}^{\prime}, h\right) \mathrm{d} \mathbf{r}^{\prime}
$$

where $w(\mathbf{u}, h)$ is an interpolation kernel function with the following properties:

$$
\begin{gathered}
\int w(\mathbf{u}, h) \mathrm{d} \mathbf{u}=1 \\
w(\mathbf{u}, h) \underset{h \rightarrow 0}{\stackrel{\delta}{\longrightarrow}}(\mathbf{u}) \\
w(\mathbf{u}, h)=0 \text { if }|\mathbf{u}|>h
\end{gathered}
$$

where $h$ is the smoothing length. There are infinitely many possible kernels. The following manipulation, which does not change the value of integral (11), is a useful 
one:

$$
\langle A(\mathbf{r})\rangle=\int \frac{A\left(\mathbf{r}^{\prime}\right)}{\rho\left(\mathbf{r}^{\prime}\right)} w\left(\mathbf{r}-\mathbf{r}^{\prime}, h\right) \rho\left(\mathbf{r}^{\prime}\right) \mathrm{d} \mathbf{r}^{\prime} .
$$

Divide the body into $\mathrm{N}$ small volume elements. The mass of the $i$-th element is $m_{i}$ and the mass center is $\mathbf{r}_{i}$. Utilizing the quantities introduced we get integral 13 in the form

$$
\langle A(\mathbf{r})\rangle=\sum_{i=1}^{N} m_{i} \frac{A\left(\mathbf{r}_{i}\right)}{\rho\left(\mathbf{r}_{i}\right)} w\left(\mathbf{r}-\mathbf{r}_{i}, h\right) .
$$

Conservation equations of continuum mechanics for mass, momentum and energy are

$$
\begin{aligned}
\frac{\mathrm{d} \rho}{\mathrm{d} t} & =-\rho \frac{\partial u^{m}}{\partial x^{m}}, \\
\frac{\mathrm{d} U^{n}}{\mathrm{~d} t} & =-\frac{1}{\rho} \frac{\partial \sigma^{n m}}{\partial x^{m}}, \\
\frac{\mathrm{d} E}{\mathrm{~d} t} & =-\frac{\sigma^{n m}}{\rho} \frac{\partial u^{n}}{\partial x^{m}}
\end{aligned}
$$

where $\rho$ is the density, $E$ is the specific internal energy. $n, m=1,2,3$ are indices for three spatial directions. $x^{m}$ is spatial coordinate, $u^{n}, u^{m}$ are the spatial components of the velocity vector and $\sigma^{n m}$ is an element of the Cauchy stress tensor.

\section{MODEL VALIDATION}

4.1. Measurements. It is important to verify the applicability of the different mathematical models and the entire numerical approaches to simulate the soil-plow
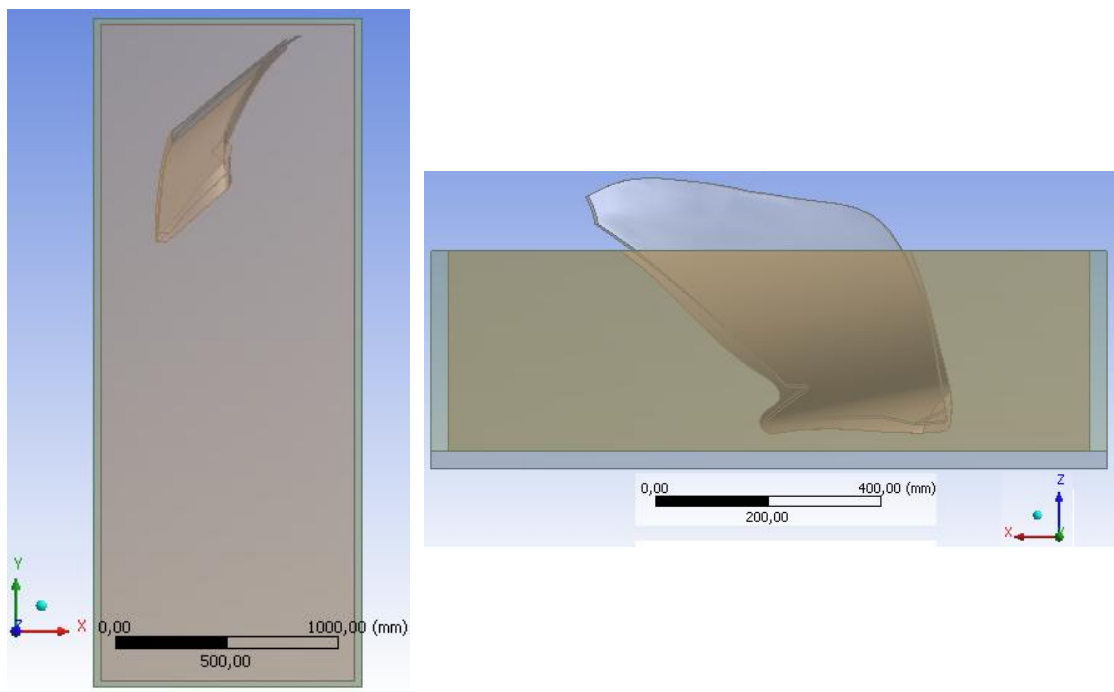

Figure 2. Experimentally studied plow moldboard 
moldboard interaction. For this reason a sufficiently well presented experimental test with the appropriate results has been chosen from the available literature. Formato et al. [12 investigated experimentally and numerically a plow moldboard and compared the results. Figure 2 shows the experimentally studied plow moldboard Formato of et al. 12 The experimental test was carried out with operating velocity $2.86 \mathrm{~m} / \mathrm{s}$ and working depth $0.3 \mathrm{~m}$. Table 2 shows the known soil properties of the field where the experimental test was carried out. The measured draft force was $4150 \mathrm{~N}$ and the total pressure was monitored at 14 different points on the surface of the plow.

Table 2. Soil properties applied in the validation of the numerical calculations from Formato el al. [10]

\begin{tabular}{|l|c|}
\hline Soil texture & Sandy loam \\
\hline \hline Sand [\%] & 81 \\
\hline Silt [\%] & 3 \\
\hline Clay [\%] & 16 \\
\hline Bulk density [kgm $\left.{ }^{-3}\right]$ & 2100 \\
\hline Moisture content [\%] & 12 \\
\hline Soil-Steel friction angle [deg] & 0.4 \\
\hline Soil-Soil friction angle [deg] & 0.6 \\
\hline
\end{tabular}

4.2. Validation of the Finite Volume CFD method. The Bingham plastic material model has been validated by experimental tests published in the literature. The necessary mechanical properties of the soil to apply the Bingham model have not been specified: for this reason the unknown parameters have been specified by parameter identification. The known draft force and the pressure values on the surface of the moldboard have been used to specify the unknown yield stress and plastic viscosity values. Several combinations of the yield stress and plastic viscosity is examined from values $\tau_{i j}=8 \mathrm{kPa}, \eta=0.1 \mathrm{~Pa}$ s to $\tau_{0}=5 \mathrm{kPa}, \eta=1 \mathrm{kPa}$ s. Table 3 contains the values of the necessary parameters applied in the Bingham model. The calculated draft force with the specified model parameters is $4176.8 \mathrm{~N}$ in case of the Bingham model with visco-plastic parameters $\tau_{0}=7.1 \mathrm{kPa}, \eta_{0}=0.1 \mathrm{~Pa}$ s. The relative error is

$$
\varepsilon_{F}=\frac{\left|F_{\text {Measured }}-F_{\text {Calculated }}\right|}{F_{\text {Measured }}} 100=0.00646=0.646 \quad[\%]
$$

between the measured and the calculated results. The value of the soil plastic viscosity seems very small compared to the measured values $\eta \approx 50-200 \mathrm{kPa}$ presented by Karmakar and Kushwaha 22. The main reason for the difference is that soil viscoplasticity values were measured for highly compacted conditions because Karmakar and Kushwaha 23 investigated flat tillage tool geometry where the soil is significantly compacted at the front region of the tillage tool. Zielinski et al. 24 report three orders of magnitude smaller plastic viscosity values $\eta \approx 0.05-1 \mathrm{kPa}$ for dry and wet soil with different kaolinite content. Plastic viscosity values of different types of soil with fall cone test has been measured by Mahajan and Budhu 25] and the results show that the soil viscosity values are in the range $\eta \approx 0.036-0.328 \mathrm{kPa}$ s similar to the results 
presented by Zielinski et al. 24]. The soil-moldboard interaction is a completely different tillage process because the soil is not compacted; it is rather loose in the case of plowing. This process further reduces the value of the visco-plastic parameters of the soil. Table 3 shows a quantitative comparison of the measured and calculated results with a simple statistical measure. Geometrical location of the control points can be seen in the second, third and fourth columns of Table 3 The following three columns show the measured and calculated pressure at those points and in the last column the relative error of the calculations is presented. Relative error of the pressure values has been calculated with the following formula

$$
\varepsilon_{P}=\frac{\left|P_{\text {Measured }}-P_{\text {Calculated }}\right|}{P_{\text {Measured }}} 100[\%]
$$

Table 3. Comparison of the measured and the calculated results

\begin{tabular}{|r|c|c|c|c|c|c|}
\hline \multirow{2}{*}{ Point } & $x[\mathrm{~m}]$ & $y[\mathrm{~m}]$ & $z[\mathrm{~m}]$ & \multicolumn{2}{|c|}{ Pressure [kPa] } & Relative error [\%] \\
\hline 5 & 0.1649 & 0.44 & 0.30 & 23.000 & 20.7768 & 10.70 \\
7 & 0.4182 & 0.68 & 0.31 & 12.000 & 12.5843 & 4.86 \\
11 & 0.1477 & 0.32 & 0.19 & 24.000 & 21.9672 & 8.47 \\
12 & 0.2884 & 0.28 & 0.09 & 28.000 & 25.0045 & 10.70 \\
13 & 0.2192 & 0.20 & 0.09 & 30.000 & 27.0302 & 9.90 \\
14 & 0.1460 & 0.12 & 0.09 & 32.000 & 33.6126 & 5.03 \\
\hline
\end{tabular}

4.3. Smoothed-particle hydrodynamics. Figure 2 shows the computational domain with the investigated plow moldboard geometry. The greatest advantage of this method is that it is not necessary to calibrate the model if the real soil parameters are known. In this case the internal friction of soil, friction between soil and tool material, and the bulk density are known [12. Bulk modulus, shear modulus are supposed as average soil, but the cohesion is not known. Calibration of cohesion was done by the measured draft force at a given speed [12. Parameters for the SPH model are the following: coefficient of internal friction is $\mu_{\text {soil }}=0.3$ coefficient of friction between soil and steel $\mu_{\text {soil-steel }}=0.4$, bulk density of soil $\rho_{p}=2100 \mathrm{kgm}^{-3}$, bulk modulus $E_{\text {soil }}=9.57 \times 10^{6} \mathrm{~Pa}$, shear modulus $G_{\text {soil }}=2.96 \times 10^{6} \mathrm{~Pa}$, cohesion $c=100 \mathrm{~Pa}$, particle size $h=0.3 \mathrm{~m}$. Modeling the soil-tool interaction with the SPH model a $3 \mathrm{~m}$ long segment of a soil bin has been used which was a width of $1.2 \mathrm{~m}$, and the height was $0.385 \mathrm{~m}$. A small gap is needed between the granular material (SPH model) and the solid surface of the investigated plow in the SPH discretization which can be seen in Figure 4. Boundary conditions were not specified at surface of SPH model in AUTODYN. A $30 \mathrm{~mm}$ thick walls were modelled at the bottom and four sides of the SPH soil model. 
4.4. Discrete element method. Data for the problem. A clump of two spherical particles having $5 \mathrm{~mm}$ radius, with $8 \mathrm{~mm}$ distance between their centers was used as soil particle model. The particle size distribution in the final granular assembly modeling the soil was randomly changing between $5 \mathrm{~mm}$ and $7.5 \mathrm{~mm}$ radii. The following micromechanical parameters were calibrated for the description of the interaction between the soil particles, and between the soil particles and the tool (index $p$ means soil particle, index $t$ means tool material): Poisson's ratio $\nu_{p}=02, \nu_{t}=0.3$, shear modulus $G_{p}=10^{7} \mathrm{~Pa}, G_{t}=8 \times 10^{9} \mathrm{~Pa}$, density $\rho_{p}=3200 \mathrm{~kg} / \mathrm{m}^{3}, \rho_{t}=7500$ $\mathrm{kg} / \mathrm{m}^{3}$. Coefficient of restitution: $C_{r, p-p}=C_{r, t-p}=0.4$, coefficient of static friction $\mu_{p-p}=0.9, \mu_{p-t}=0.35$, coefficient of rolling friction $\mu_{r, p-p}=0.015, \mu_{r, p-t}=0.01$. For the bonded soil model we used: normal stiffness $S_{n}=10^{9} \mathrm{Nm}^{-3}$, shear stiffness $S_{t}=10^{9} \mathrm{Nm}^{-3}$, critical normal stress $\sigma_{\max }=1.5 \times 10^{6} \mathrm{~Pa}$, critical shear test $\tau_{\max }=9 \times 10^{5} \mathrm{~Pa}$, bonded disk radius $R_{B}=0.005 \mathrm{~m}$.

Draft force prediction. For modeling the soil-tool interaction, the discrete element model of a $1 \mathrm{~m}$ long segment of a soil bin was used. The width of the duct was the double of the tool width, and in this direction a periodic boundary condition is used to simulate the effect of the neighboring soil particles. The use of periodic boundary in the direction perpendicular to the tool displacement direction enabled us to reduce effectively the size of the simulation domain, and in this way the simulation time requirements. The thickness of granular assembly beneath the tool was the same as the height of the tool itself. The simulation time step was set to be $25 \%$ of the Rayleigh time step, using the material parameters listed above. The simulation started with filling the calculation domain of the soil bin model with particles using

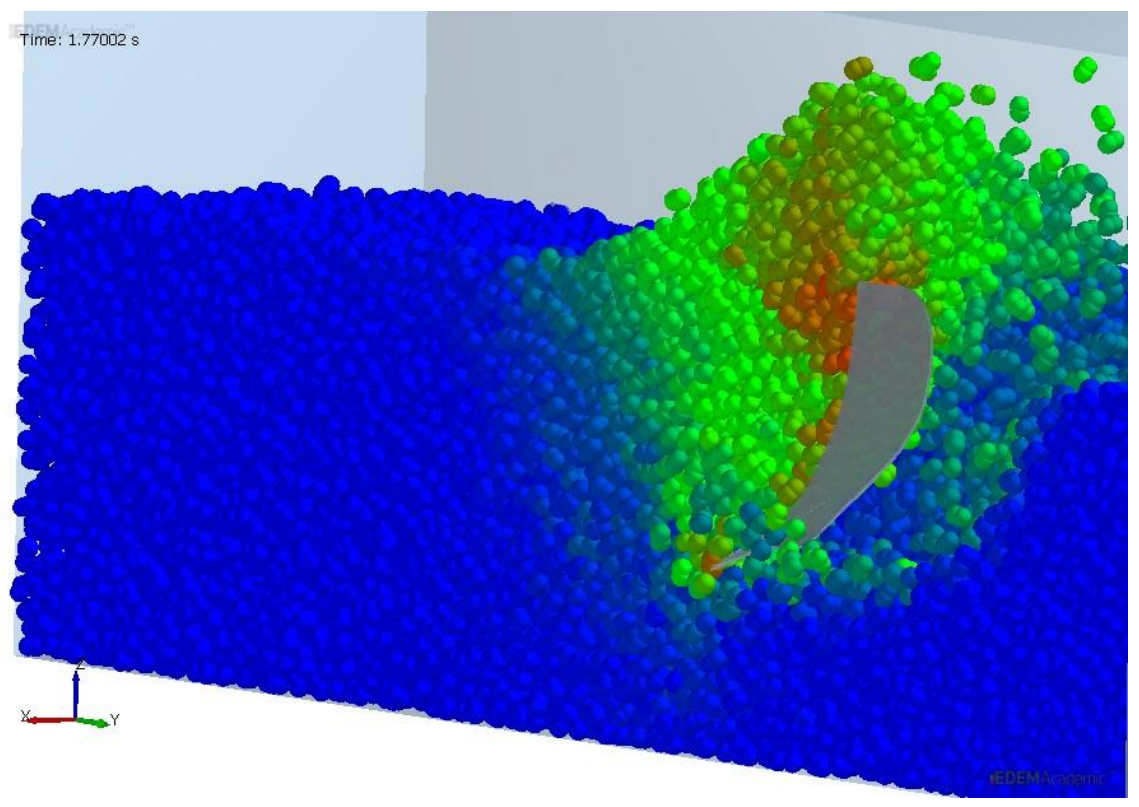

Figure 3. Soil-tool interaction DEM model, the tool geometry and the model bin are shown here, the boundary is periodic in $x$ direction. 
dropping method. We filled the bin with particles by letting their gravitational sedimentation form a randomly generated assembly initially filling the whole bin, and then settling down to a state when it was filling approximately one third of it (Figure 3). One second after this sedimentation process (this time needed to get the particles to be in a state of rest), we "turned on" the bonding model, gluing together the soil particles. After this, the tillage tool started its way through the bin. Only the forces acting in the middle third section of the model bin were used to evaluate the average draft force value, to avoid the effect of the end wall, and the effect of increasing the number of contacts during the tool's immersion in the soil.

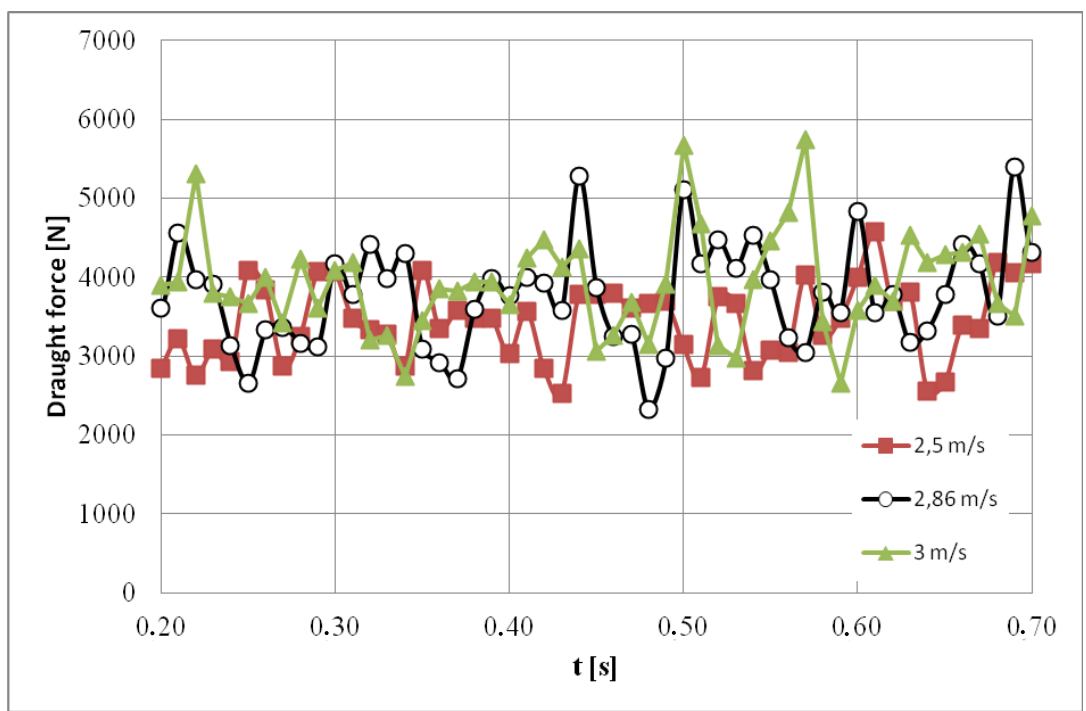

Figure 4. Sample diagram of measured and simulated draft forces

The simulated draft forces showed significantly higher deviation than the measured ones. This phenomenon is mainly caused by the large particle size used because of the restrictions in computer simulation capabilities. Sample simulation results can be seen in Figure 4. It can be seen, that the simulated draft forces are in good agreement with the measured ones [12]. The average plow forces related to different velocities are as follows:

\begin{tabular}{|c|c|}
\hline$v[\mathrm{~m} / \mathrm{s}]$ & $F[\mathrm{~N}]$ \\
\hline \hline 2.00 & 3068.474 \\
\hline 2.50 & 3445.344 \\
\hline 2.86 & 3842.261 \\
\hline 3.00 & 3930.810 \\
\hline 3.50 & 4264.764 \\
\hline
\end{tabular}




\section{RESUlts}

5.1. Finite volume method. Figures 5 and 6 show some stages of the turnover of a furrow slice. It can be clearly seen that if the grid resolution is sufficiently fine in that case the soil-air interface is sharply recognizable.

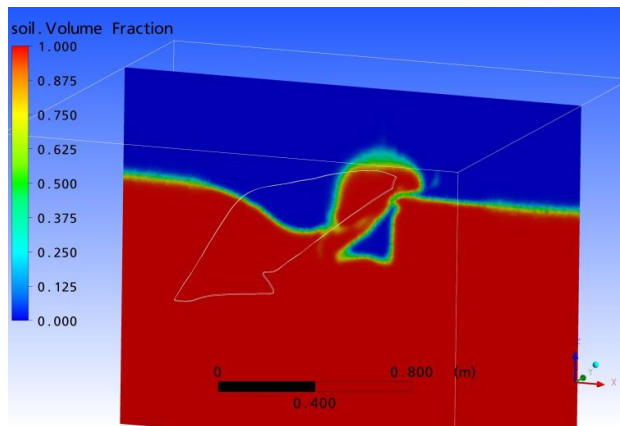

(a)

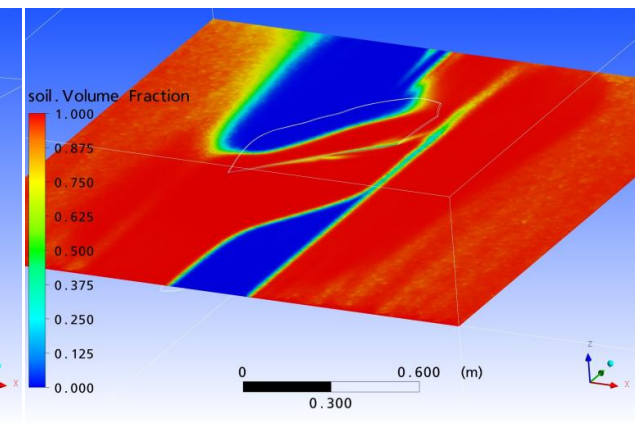

(b)

Figure 5. Perspective view of the furrow and the turn over of the furrow slice

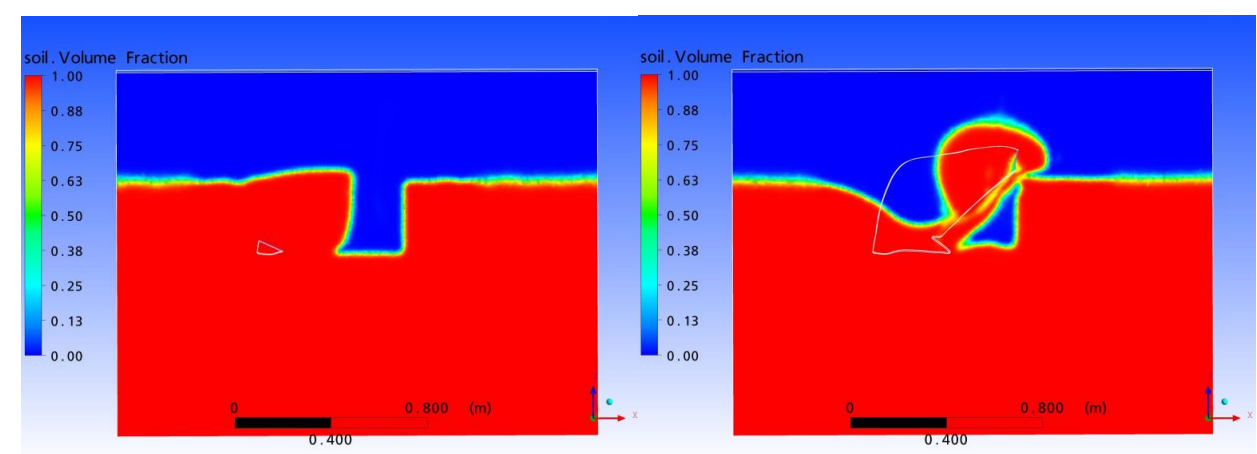

Figure 6 . Front view of the furrow and the turn over of the furrow slice
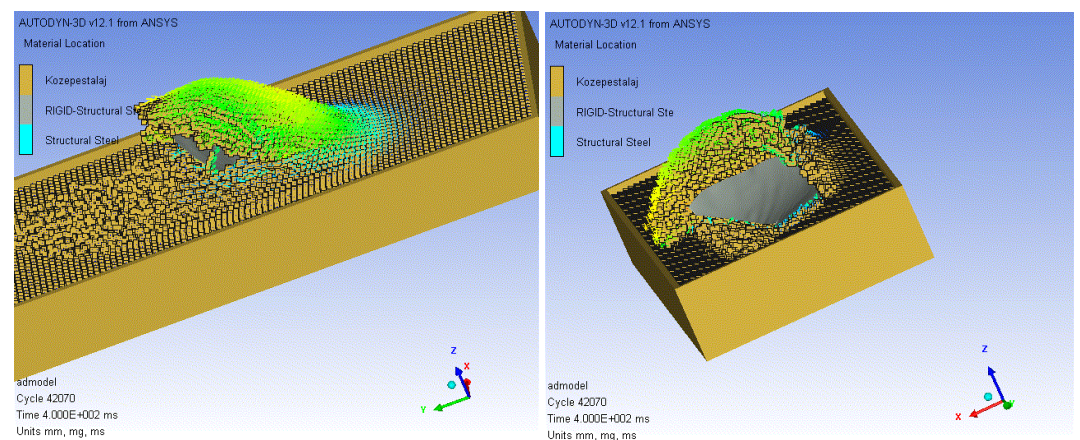

Figure 7. Perspective view of the flow movement in the case of SPH simulation 
5.2. SPH method. Figure 7 shows the simulated deformation of SPH model of soil with $30 \mathrm{~mm}$ particle size. Predicted deformations are more realistic than DEM. Soil particles are much smaller than in the other two methods. Hence the SPH particles (which are not real particles) are better for prediction of deformations.

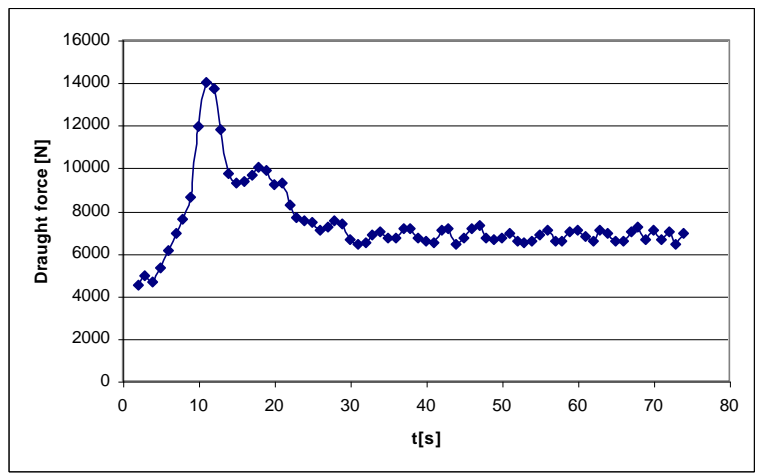

Figure 8. Sample diagram of measured and simulated draft forces

The real soil parameters are used in this method so it is not necessary to calibrate the model. But the uncalibrated model predicts higher draft forces than those measured. Another advantage of this method is prediction of transient stadium with local peak (Figure 8).

\section{Conclusions}

Three different mathematical modeling and numerical computation approaches have been applied to investigate the soil-tool interaction for a moldboard plow. Comparison made between the different modeling approaches to get information about the efficiency of the applied models was validated by an experimental test available from the literature. The following general conclusions can be drawn:

1. It can be stated that a validation with only a measured draft force value of a soil tillage tool is not enough to calibrate the different models correctly because of the complex behavior and structure of the soil. The models generally need a lot of measured parameters that have to be calibrated before the computation. For this reason it can be suggested that more information is needed to validate a numerical model than a single draft force value. For example pressure distribution on the surface of the tillage tool where the soil interacts with the tool or local velocity measurements of the flow field of the soil around the tillage tool can be useful additional information for model validation purposes.

2. Area of modeling possibilities: The finite volume CFD method effectively describes the flow behavior of granular materials that can be modeled by nearly spherical particles. Shortcomings: it is only capable of modeling the flow behavior of granular material which consists of nearly spherical particles with 
smaller particle size than the conventional size of agricultural granular products (wheat, maize, barley, etc. The finite volume CFD method cannot be applied such granular materials where particle geometry significantly differs from spherical shape like stringy crops. It is difficult to specify correctly the physical properties of the modeled granular material. The proper value of the plastic viscosity and yield stress cannot be determined precisely because these properties are pressure dependent. For this reason the plastic viscosity and yield stress cannot be characterized with a constant value for computing soil flow. Computational requirements: the finite volume method needs significantly larger amount of computational resources, mostly operative RAM simulating sufficiently detailed interface between the soil-air and soil-tillage tool interaction compared to the other two investigated methods. The necessary calculation time takes two or even three times longer than the SPH-FEM and the DEM method.

3. Area of modeling possibilities: The Smoothed-Particle Hydrodynamics (SPH) method can be used effectively with flow cases where the flow field has a lot of separated regions. The SPH method is also sensitive to the particle size, which has to be chosen properly to avoid physically unacceptable oscillations of the solution.

4. Area of modeling possibilities: The Discrete Element Method can be considered the most widely applicable method to describe the flow process of granular materials, because it can use almost any kind of particle geometry and from physical modeling point of view it is the most realistic description of a granular material. For this reason the discrete element method is capable of effectively modeling the soil-tool interaction process. DEM is capable of simulating draft force, but the pressure distribution cannot be easily modeled by using DEM. The simulated draft forces showed significantly higher deviation than the measured ones. This phenomenon is mainly caused by the large particle size used because of the restrictions in computer simulation capabilities. There is no guarantee that the calibrated micromechanical parameters are the only ones resulting the desired draft force values. Shortcomings: the greatest problem with this modeling approach is that current computational resources cannot calculate with the same amount of particle numbers contained in a real granular material. The most difficult is the proper calibration of the DEM model. There are 12 parameters (including time step) to be properly calibrated for determining the micromechanical parameters describing the mechanical behavior of the particle assembly. By using the particle-rigid body interaction model, the reaction forces acting on the tool are not taken into account as excitations generating for example the vibration of the tool, which can cause errors in the modeling results, especially in case of easily vibrating tools. Computational requirements: computational time requirement is approximately 5 hours on a dual core $\mathrm{P} 8600-2.4 \mathrm{GHz}$ 8GB RAM computer (this relatively short computational time was the result of low particle shear modulus). The calibration of micromechanical parameters is a significantly more time consuming procedure, because of the need for a large number of simulations to be done. 
Acknowledgement. This paper was supported by the János Bolyai Research Scholarship of the Hungarian Academy of Sciences.

\section{REFERENCES}

1. Karmakar, S., Kushwaha, R. L. and Lague, C.: Numerical modeling of soil stress and pressure distribution on a flat tillage tool using computational fluid dynamics. Biosystems Engineering, 97(3), (2007), 407-414.

2. Godwin, R.: A review of the effect of implement geometry on soil failure and implement forces. Soil \& Tillage Research, 97, (2007), 331-340.

3. Godwin, R. J. and O'Dogherty, M. J.: Integrated soil tillage force prediction models. Journal of Terramechanics, 44, (2007), 3-14.

4. Onwualu, A. P. and Watts, K. C.: Draught and vertical forces obtained from dynamic soil cutting by plane tillage tools. Soil \& Tillage Research, 48, (1998), 239-253.

5. Chandler, H. W.: The use of nonlinear fracture mechanics to study the fracture properties of soils. Journal of Agricultural Engineering Research, 29, (1984), 321-327.

6. Aluko, O. B. and Chandler, H. W.: A fracture strength parameter for brittle agricultural soils. Biosystems Engineering, 93(2), (2006), 245-252.

7. Kocher, M. F. and Summers, J. D.: Wave propagation theory for evaluating dynamic soil stress-strain models. Transactions of the ASAE, 31(3), (1985), 683-691.

8. Rosa, U. A. and Wulfsohn, D.: Constitutive model for high speed tillage using narrow tools. Journal of Terramechanics, 36, (1999), 221-234.

9. Mounzen, A. and NeméNyi, M.: Tillage tool design by the finite element method-part 1: finite element modeling of soil plastic behavior. Journal of Terramechanics, 72, (1999), $37-51$.

10. Elijah, D. L. and Weber, J. A.: Soil failure and pressure patterns for flat cutting blades. Transactions of the ASAE, 14(4), (1971), 781-785.

11. Fielke, J. M.: Finite element modeling of the interaction of the cutting edge of tillage implements with soil. Journal of Agricultural Engineering Research, 74, (1999), 91-101.

12. Formato, A., Faugno, S. and Paolillo, G.: Numerical simulation of soil-plough mouldboard interaction. Biosystems Engineering, 92(3), (2005), 309-316.

13. Cundall, P. A. and Strack, O. D. L.: A discrete numerical model for granular assemblies. Geotechnique, 29(1), (2012), 47-65.

14. DemSolutions: Edem User's Guide. 2012.

15. Monaghan, J. and Gingold, R.: Smoothed particle hydrodynamics. Mon. Not. Roy. Astr. Soc., 181, (2012), 375-389.

16. Hayhurst, C. J., Clegg, R. A., Livingstone, I. H. and Francis, N. J.: The application of sph techniques in AUTOSDYN-2d to ballistic impact problems. 16th International Symposium on Ballistics, San Francisco, CA, USA, 1966.

17. Chakraborty, S. and Shaw, A.: A pseudo-spring based fracture model for SPH simulation of impact dynamics. International Journal of Impact Engineering, 58, (2013), $84-95$.

18. Yang, Q., Jones, V. and McCue, L.: Free-surface flow interactions with deformable structures using an SPH-FEM mode. Ocean Engineering, 55, (2012), 136-147. 
19. Bui, H. H., SAko, K. and Fukagawa, R.: Numerical simulation of soil-water interaction using smoothed particle hydrodynamics (SPH) method. Journal of Terramechanics, 44, (2007), 339-346.

20. Lu, Y., WANG, Z. and ChONG, K.: A comparative study of buried structure in soil subjected to blast load using $2 \mathrm{~d}$ and $3 \mathrm{~d}$ numerical simulations. Soil Dynamics and Earthquake Engineering, 25, (2005), 275-288.

21. Calamaz, M., Limido, J., Nouari, M., Espinosa, C., Coupard, D., Salaün, M., Girot, F. and Chieragatti, R.: Toward a better understanding of tool wear effect through a comparison between experiments and SPH numerical modelling of machining hard materials. Int. Journal of Refractory Metals $\&$ Hard Materials, 27, (2009), 595-604.

22. Karmakar, S. and Kushwaha, R. L.: Dynamic modeling of soil-tool interaction: An overview from a fluid flow perspective. Journal of Terramechanics, 43(4), (2005), 411425.

23. Karmakar, S. and Kushwaha, R. L.: Development and laboratory evaluation of a rheometer for soil visco-plastic parameters. Journal of Terramechanics, 44, (2007), 197204.

24. Zielinski, M., Dembicki, E. and Monnet, J.: Studying slow displacements of the natural slopes using particle image velocimetry method. 18. Congres Francais de Mecanique, Grenoble, August 27-31, 2007.

25. Mahajan, S. P. and Budhu, M.: Shear viscosity of clays to compute viscous resistance. The 12th International Conference of International Association for Computer Methods and Advances in Geomechanics (IACMAG) 1-6 October, 2008, Goa, India, pp. 15161523. 